\title{
FORENSIC IMAGING OF GLASS GOBLET USING CHALK AND SUblimation SPRAY
}

\author{
Jiri Sevcik, Milan Adamek \& Jan Valouch
}
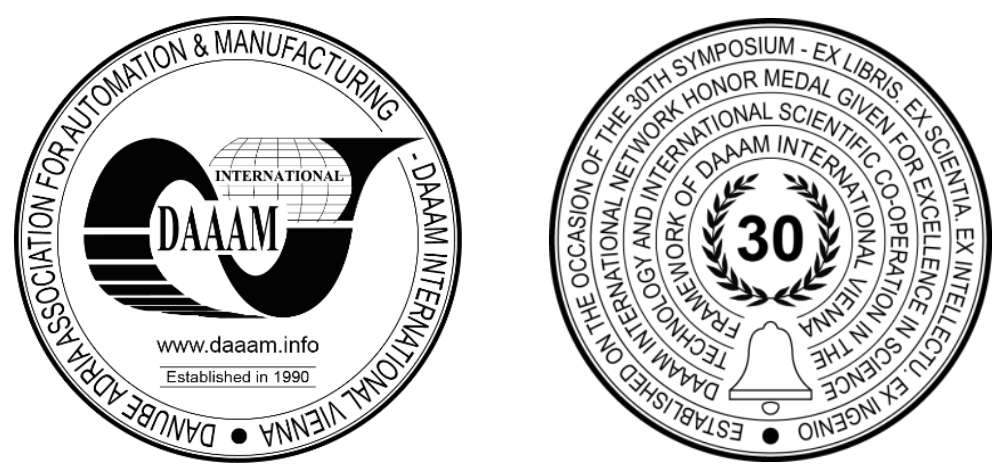

This Publication has to be referred as: Sevcik, J[iri]; Adamek, M[ilan] \& Valouch, J[an] (2020). Forensic Imaging of Glass Goblet Using Chalk and Sublimation Spray, Proceedings of the 31st DAAAM International Symposium, pp.05300534, B. Katalinic (Ed.), Published by DAAAM International, ISBN 978-3-902734-29-7, ISSN 1726-9679, Vienna, Austria

DOI: $10.2507 / 31$ st.daaam.proceedings.073

\begin{abstract}
3D imaging represents fast evolving method, which finds contribution in variety of research areas. One of possible applications could be examined in conjunction with forensic research. This paper is addressing contemporary problem of scanning highly reflective or translucent surfaces, since its close relation to fast virtual crime scenario reconstruction viability. The virtual crime scenario reconstruction methodology is proposed and the initial testing of translucent surface scanning, by utilisation of photogrammetry technique and surface enhancement by non-destructive chalk and sublimation spray.
\end{abstract}

Keywords: 3D imaging; scanning; photogrammetry; forensic science;

\section{Introduction}

The utilisation of spatial scanning and visualisation methods within growing number of forensic applications. Moreover, new or innovated methods development is realized throughout this interdisciplinary connection. The main goal of these evolution is potential to reduce bias related to crime investigation and culprit proving process. Complexity of individual methods utilisation has predestined them primarily for cases where hard crime was committed. Forensic archaeology, anthropology, footprint reconstruction, odontology or crime scene investigation and demonstration on the court could me mentioned as an examples. [1]. The 3D reconstruction process is characterized by several steps, its complexity is depended on level of detail in which should be final model computed [2]. Desired level of detail is in most cases directly influenced by purpose of reconstruction. The objective of $3 \mathrm{D}$ reconstruction is then determined by competent authority, which could vary across the particular countries. Individual forensic sub disciplines could be divided categories based on their objective, relevant publications related to particular applications could be sorted in same way $[1]$.

\section{1. $3 D$ crime scene reconstruction}

Reflective scanning techniques utilisation for crime scene documentation, analysis and presentation has been evaluated in last two decades and already has been implemented to practice. The absence of spatial distortion is indisputable advantage of 3D data gathering techniques in comparison to 2D data acquired by standard photography. 
Main limitation for higher contribution of 3D scanned data has been their processing. However, with the evolution of hardware and software, the process of using scanned spatial data in the form of 3D reconstructions is becoming more accessible and is being used regularly by police forces to document the crime scene [3]. Empirical research focuses on comparing the accuracy of crime documentation through original and modern techniques. For example, a comparison of measurements with a conventional tape measure and photogrammetry based on images from a $360^{\circ}$ camera. The manual method shows greater accuracy in aiming specific dimensions, while the software tool has a smaller deviation of repeated measurements of the same distance [4].

Another example is the perspective application of photogrammetry to document the location of a violent crime. It was a combination of several scanning devices. Overview source images were captured using a drone, and detailed terrestrial images captured by a digital camera were combined to obtain 3D models of large space and detail at the same time. The study emphasized the use of reference targets to link photographs from different sources and positions [5]. Another study points to the possibility of combining 3D scanned data with shots from a camera surveillance system, or photographs of eyewitnesses, such data can be used to determine the position or further profiling of offenders [3].

The simulation of a bus explosion was the subject of another scientific article. The entire space, including, was successfully 3D reconstructed by means of photogrammetry, which was used for subsequent detailed crime scene analysis [6]. And many other examples of applications focused on 3D reconstruction of crime scenes and traffic accidents using photogrammetry [7] and 3D reconstruction of incidents where firearms were used [8]. A specific focus on 3D scans of weapons at the crime scene and their subsequent reconstruction and evaluation of use at the crime scene, [3] was tasked with a case study, which pointed out the possibility of using $3 \mathrm{D}$ reconstruction of the crime scene to analyze the evidence present in the scene.

\section{Problem formulation}

Scale of possible applications was described within previous section, however all of them have to challenge similar difficulties related to individual scanning techniques. Experimental multimodal data acquisition of room utilising terrestrial laser scanning and photogrammetry method has been used as a demonstrative example of negative factors, which influence results. This 3D reconstruction is notated as K001 for purposes of this research paper. The overall quality of reconstructed model was relatively good, but particular parts was defected, as you can see on Fig. 1.

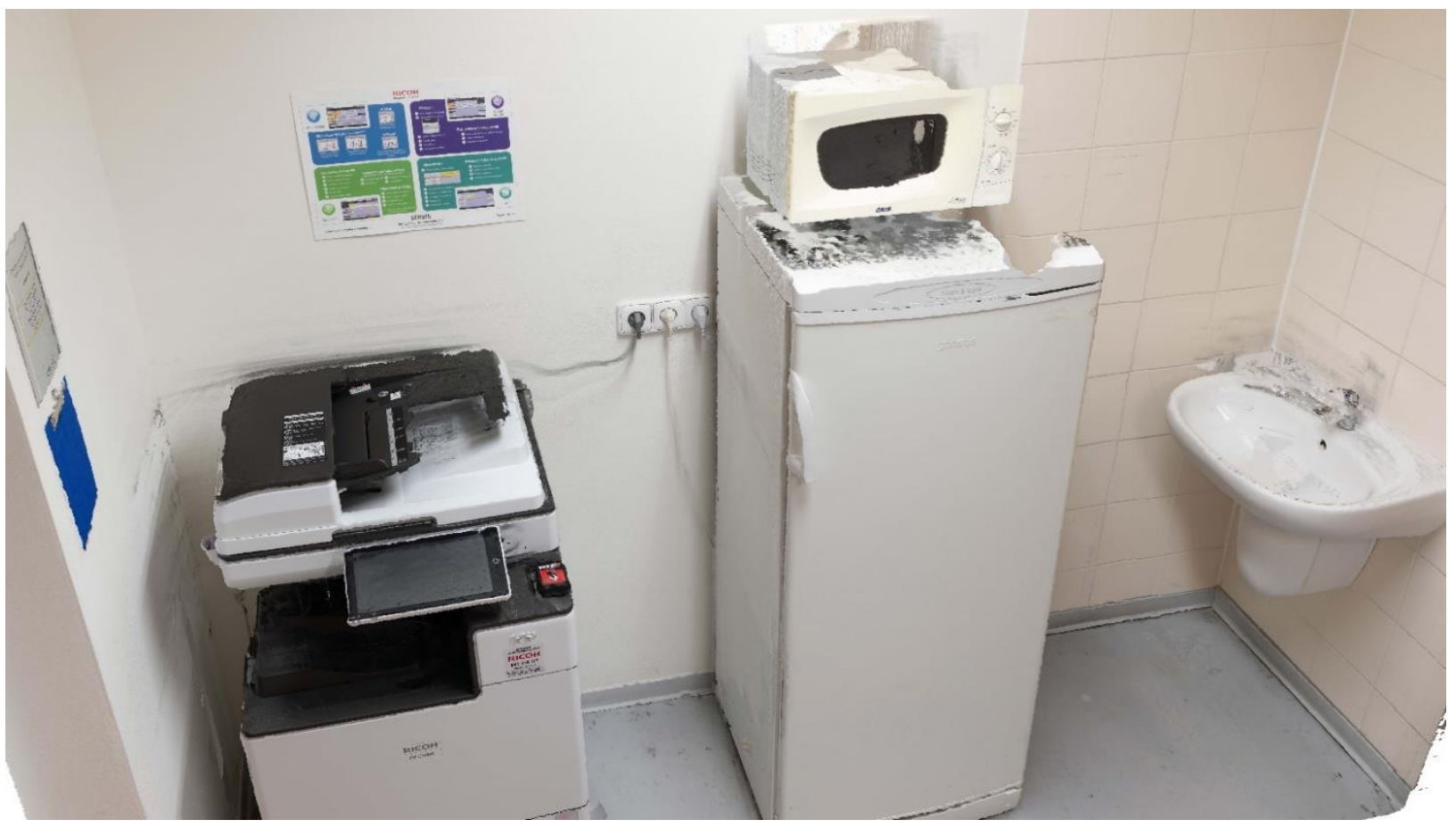

Fig. 1. Defected parts of model K001

\section{Methods of noncontact reflective scanning}

Advances in active and passive surface scanning methods are the driving force behind many related disciplines. The direct impact can also be observed in the area of 3D reconstruction of the crime scene. The use of the principle of the reflected beam of electromagnetic radiation makes it possible to obtain information about the physical structure of exposed surfaces, which can be achieved completely contactless, which is an indisputable advantage of this method in the case of documenting evidence or the entire crime scene. 
However, the quality of the obtained data is affected by the properties of the exposed surfaces and the conditions under which the scanning is performed. Properties such as roughness, humidity, shape, temperature, which can affect the reflectivity of the scanned surface, have a direct impact on the quality of the scan. Another important aspect is the availability of the scanned surface, which can be a limiting factor for the use of some methods. The main advantages of reflective techniques include short scanning time, relatively low cost of hardware and software equipment and the ability to ensure high quality scanned data. [9] Active scanning methods usually have their own source of electromagnetic radiation, the beam of this radiation is directed at the surface of the scanned body, which is then reflected back into the sensor. Passive methods do not have their own energy source, they only receive and process sources which occurs within the scanned area. The basic requirement in this case is to capture at least two images with an overlap of at least $80 \%$. When taking single pictures, either the sensor moves or the surface to be scanned moves. While the surface scanning process itself is a relatively simple task, the subsequent processing of data and the creation of 3D models already requires a high degree of expertise and knowledge of many software tools.

Currently, the following reflexive scanning techniques are used in the field of forensic sciences:

- laser triangulation,

- laser time of flight,

- structured light,

- photogrammetry.

Each of these methods has its advantages and disadvantages, which predestines them for use in a variety of applications. A promising approach is to use multiple methods for the same purpose, while increasing the likelihood of minimizing negative properties. However, this advantage further complicates the subsequent processing of spatial data that comes from multiple sources.

\subsection{Photogrammetry}

Photogrammetry technique can be implemented by means of one or more cameras and falls into the category of passive triangulation, as it has no source of radiation of its own. The single camera variant is also referred to as Structure from Motion. This method works on the principle of photographing an object of interest or space, while the minimum mutual overlap of images must be observed, which should ideally be at least $80 \%$. In the case of one camera, self-calibration is always used, as the camera position is calculated using algorithms only from the recorded data. The second version, using the same principle, uses a system of at least two cameras, which can be calibrated to each other or can also use selfcalibration. [9]

Hardware simplicity combined with high accuracy motivates empirical testing in many fields. [10] The undeniable advantage of this method in relation to the digital reconstruction of BI scenarios is the ability to generate a photorealistic level of surface texture, [4] which has been verified in several case studies. [11] The disadvantage is the high sensitivity to the condition of the scanned surfaces. Reflective and wet surfaces are especially problematic, but also hair growth. [12] The documentation of smooth surfaces can be just as demanding, which was verified on an example study of pocket knife photogrammetry. [11] All these negative phenomena have a common denominator, which is the illumination of the scanned surface. This affects both the accuracy of the skeleton and the texture of the object.

The technique can also be used for measurement, but it requires the placement of a reference object in the scanned scene and then determine the scale of the entire model according to its dimensions. The accuracy of measurement using industrial photogrammetry reaches an accuracy of up to $0.01 \mathrm{~mm}$. However, with increasing accuracy, the demand for the quality of the scanning device increases and the maximum size of the scanned object decreases. Architectural and engineering photogrammetry achieves a maximum measurement accuracy of $0.1 \mathrm{~mm}$. The main advantage of these devices compared to competing methods is price, but at the expense of lower measurement accuracy and the need to deal with many negative external phenomena.

\section{Glass goblet scanning experiment}

An evaluation of influence of sublimation and chalk spay surface application on quality of 3D reconstruction, using photogrammetry is the goal of experimental part of this research paper. Sprays are usually utilised to create appropriate surface for laser scanning. Two reconstructions of same model of goblet was realized. Acquisition of pictures was done by mirrorless camera Sony Alpha A3R. Camera setting was chosen according requirements of the method as you can see on Table. 1. Software tool Capturing reality was incorporated for alignment of images and following 3D reconstruction. Two models of goblets was reconstructed as a result of an experiment. Final models was defected as you can see in Fig. Nonetheless scanning inaccuracies could be caused by several factors. Inappropriate camera positioning, camera to object setup, incorrect amount of aerosol on the surface of the goblet and others. These problems should be addressed in future research. Moreover, utilisation of individual spray have revealed different results. Chalk spray has provided better surface coverage and mark the possible way of next research steps. Possible modification could be done also in case of camera to object setup, where rotational basement should be used with upright illumination system. 


\begin{tabular}{|l|l|}
\hline Picture dimension (pixel) & $7952 \times 5304$ \\
\hline Color depth (bit) & 24 \\
\hline Aperture number & $\mathrm{f} / 13$ \\
\hline Exposure time (sec) & 6 \\
\hline Focal lenght (mm) & 55 \\
\hline Number of pictures & 1505 \\
\hline Time to take picture approximation (sec) & 30 \\
\hline
\end{tabular}

Table 1. Image acquisition settings

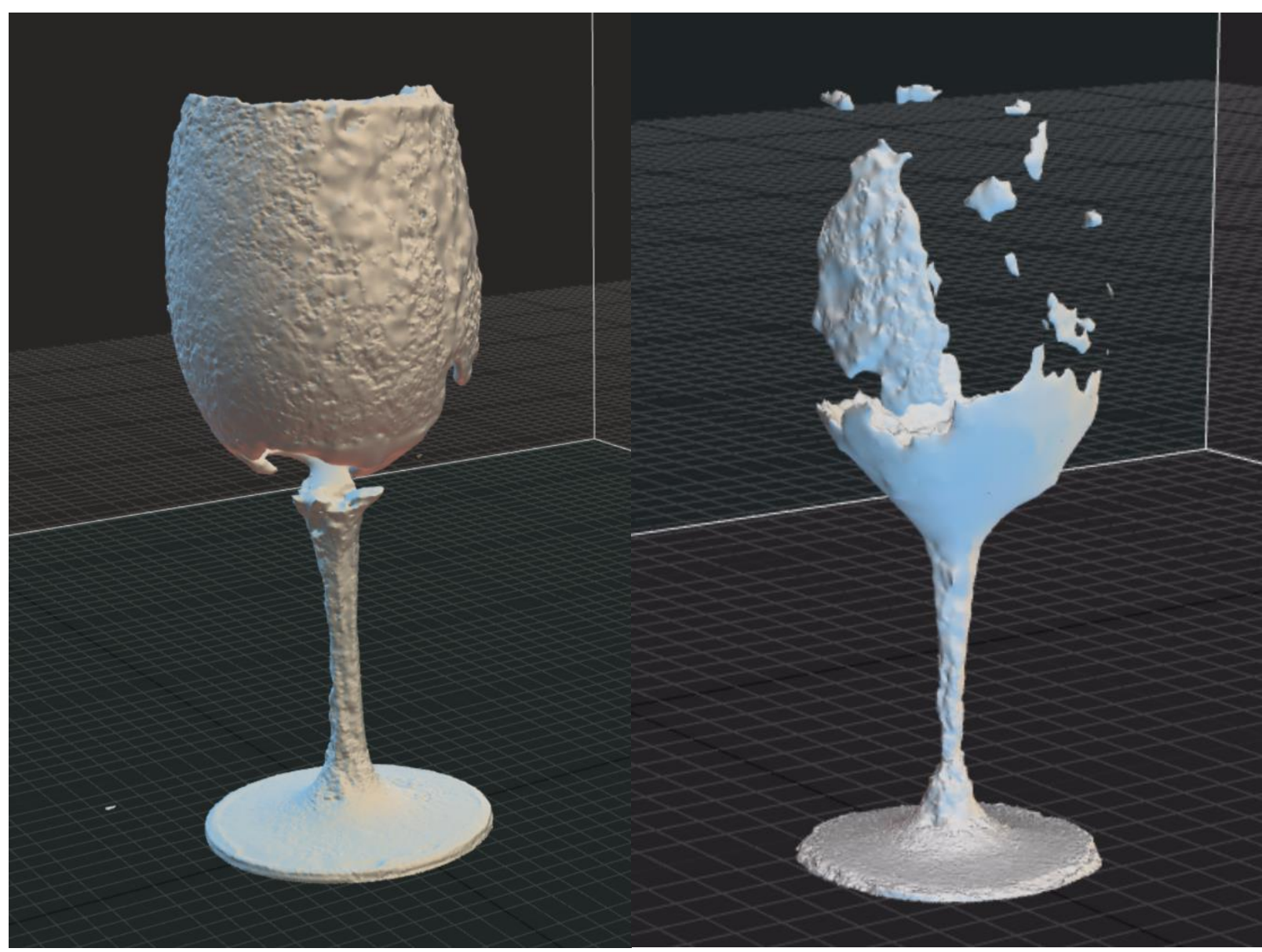

Fig. 2. Reconstruction of goblet using chalk spray on the right and sublimation spray on the left side

\section{Conclusion}

Main goal of the experimental part of this research paper was evaluation of two different types of sprays for enhancing glass materials to be able to scan through method photogrammetry. Results have shown that chalk spray has higher potential to fulfil requirement for possible further experiments. Although the main effect of sprays is intended for laser scanning techniques, we could say, that it has also positive influence in relation of photogrammetry method. The fact is, that spray surface application disallow texture scan, which is the main advantage of evaluated method in case of multimodal scanning. The results also showed, that both sprays has not provide models, which could be directly imported into crime scene reconstruction. Moreover, the further processing is needed, for the practical application of proposed method, which significantly increase the time of whole scene reconstruction process.

Photogrammetry of different shapes of glasses with improved scanning setup is a plan for further experiments, next to evaluation of spray influence in multimodal scanning. Moreover, repetitive image acquisition process with different setting should be done to verify the authenticity of results obtained. Technique evaluated in this research paper will be utilised in particular cases, such as the areas where the crime could be potentially committed. Scanning of extraordinary reflective surfaces within rooms, buildings or even exterior spaces will be the following goal of future research. 


\section{Acknowledgments}

This work was supported by the Ministry of Education, Youth and Sports of the Czech Republic within the National Sustainability Program Project No. LO1303 (MSMT-7778/2014) and also by the European Regional Development Fund under the project CEBIA-Tech ED2.1.00/03.0089 and by the Internal Grant Agency of Tomas Bata University under the project No. IGA/CebiaTech/2020/003.

\section{References}

[1] Rachael M. Carew, David Errickson, Imaging in forensic science: Five years on, Journal of Forensic Radiology and Imaging, Volume 16, 2019, Pages 24-33, ISSN 2212-4780,

[2] Sieberth, Till \& Dobay, Akos \& Affolter, Raffael \& Ebert, Lars. (2018). Applying virtual reality in forensics - a virtual scene walkthrough. Forensic Science, Medicine and Pathology. 15. 1-7. 10.1007/s12024-018-0058-8.

[3] Raneri D., Enhancing forensic investigation through the use of modern three-dimensional (3D) imaging technologies for crime scene reconstruction, Aust. J. Forensic Sci. (2018) 1-11,

[4] Sheppard K., Cassella J.P.,Fieldhouse S., A comparative study of photogrammetric methods using panoramic photography in a forensic context, Forensic Sci. Int. 273 (2017) 29-38,

[5] Urbanová P.,Jurda M.,Vojtíšek T, Krajsa J., Using drone-mounted cameras for on-site body documentation: 3D mapping and active survey, Forensic Sci. Int. 281 (2017) 52-62, https://doi.org/10.1016/j.forsciint.2017.10.027.

[6] Villa C., Hansen N.F., Hansen K.M., Hougen H.P., Jacobsen C., 3D reconstructions of a controlled bus bombing, J. Forensic Radiol. Imaging 12 (2018) 11-20,

[7] Michienzi R., Meier S., Ebert L.C., Martinez R.M., Sieberth T., Comparison of forensic photo-documentation to a photogrammetric solution using the multicamera system "Botscan”, Forensic Sci. Int. 288 (2018) 46-52,

[8] P.JD. Ward, F.P. Sheridan, 3D reconstruction of shooting incidents using laser scanning and computer modeling, Proceedings of the AAFS, 2018, p. 536.

[9] Sansoni G.,Trebeschi M., Docchio F., State-of-the-art and applications of 3D imaging sensors in industry, cultural heritage, medicine, and criminal investigation, Sensors 9 (1) (2009) 568-601,

[10] Carlton C.D., Mitchell S., Lewis P., Preliminary application of Structure from Motion and GIS to document decomposition and taphonomic processes, Forensic Sci. Int. 282 (2018) 41-45,

[11] Peterson, E.B., M. Klein and R.L. Stewart (2015). Whitepaper on Structure from Motion (SfM) Photogrammetry: Constructing Three Dimensional Models from Photography. Report ST-2015-3835-1 for the U.S. Bureau of Reclamation, Research and Development Office, U.S. Bureau of Reclamation, Denver, Colorado.

[12] Ebert L., Flach P., Schweitzer W., Leipner A., Kottner S., Gascho D., Breitbeck R., Forensic 3D surface documentation at the Institute of Forensic Medicine in Zurich - workflow and communication pipeline, J. Forensic Radiol. Imaging 5 (2016) 1-7,

[13] Melichar M., Kubátová D., Kutlwaše J., (2016). CMM measuring cycle and human factor. Proceedings of the 27 ${ }^{\text {th }}$ DAAAM International Symposium. ISBN 978-3-902734-08-

[14] Kubátová, D; Melichar, M \& Kutlwaser, J (2017). Impact of Stylus Size in Roughness Measurement, Proceedings of the 28th DAAAM International Symposium, pp.0457-0466, B. Katalinic (Ed.), Published by DAAAM International, ISBN 978-3-902734-11-2, ISSN 1726-9679, Vienna, Austria DOI: 10.2507/28th.daaam.proceedings.064. 